\title{
Calculations of kaonic nuclei based on chiral meson-baryon coupled-channel interaction models
}

\author{
Jaroslava Hrtánková*, Jiří Mareš \\ Nuclear Physics Institute, 25068 Řež, Czech Republic \\ E-mail: hrtankova@ujf.cas.cz
}

\begin{abstract}
We report on our recent self-consistent calculations of $K^{-}$-nuclear quasi-bound states using a $K^{-}$ single-nucleon optical potential derived from 6 different chiral meson-baryon coupled-channel interaction models. They yield quite diverse $K^{-} 1 s$ binding energies and rather small $K^{-}$decay widths. Next, we supplement the $K^{-}$single-nucleon potential by a phenomenological $K^{-}$multinucleon potential introduced recently to achieve good fits to kaonic atom data [1]. We demonstrate a decisive impact of the $K^{-}$multinucleon interactions on the widths of $K^{-}$-nuclear states. The resulting widths are considerably larger than the corresponding binding energies. The only two models accepted by the analysis of Ref. [1] even do not yield any kaonic nuclear bound state in many-body nuclear systems with mass number $A \leq 40$.
\end{abstract}

55th International Winter Meeting on Nuclear Physics

23-27 January 2017

Bormio, Italy

\footnotetext{
* Speaker.
} 


\section{Introduction}

The $K^{-} N$ interaction near threshold is attractive as can be deduced from analyses of kaonic atom data and the existence of the $I=0 \pi \Sigma$ resonance $\Lambda(1405)$. This feature has encouraged experimental searches for $K^{-}$-nuclear bound states, however, the question of their binding energies and widths and even their very existence is far from being settled yet. Current microscopic description of the $K^{-} N$ interaction near threshold is provided by chiral SU(3) coupled-channel meson-baryon interaction models, namely: Prague (P) [2], Kyoto-Munich (KM) [3], Murcia (M1 and M2) [4], and Bonn (B2 and B4) [5]. These models capture the physics of $\Lambda(1405)$ and reproduce low energy $K^{-} N$ observables, including the $1 s$ level shift and width in the $K^{-}$hydrogen atom from the SIDDHARTA experiment [6].

The near threshold $K^{-} N$ scattering amplitudes in these chirally-inspired models are strongly energy dependent due to the presence of the $\Lambda(1405)$ resonance. This calls, together with density dependent in-medium modifications, for self-consistent evaluation of the $K^{-}$-nucleus optical potential relevant for calculations of $K^{-}$-nuclear states. The above chiral models describe only the $K^{-}$ absorption on a single nucleon, while $K^{-}$interactions with two and more nucleons take place in the nuclear interior as well. In fact, $K^{-}$multinucleon absorption governs the widths of deeply bound $K^{-}$-nuclear states [7]. It is thus desirable to take into account the $K^{-}$multinucleon interactions in calculations of kaonic nuclei.

In this contribution, we report on our very recent calculations of $K^{-}$-nuclear quasi-bound states using the above mentioned chiral models of meson-baryon interactions $[8,9]$. The $K^{-}$singlenucleon potential is supplemented by a phenomenological $K^{-}$multinucleon potential fitted recently to kaonic atom data for each of the chiral models separately [1]. In Section 2, we briefly introduce the kaon self-energy operator including in-medium modifications of the underlying $K^{-} N$ scattering amplitudes and demonstrate how to incorporate strong energy and density dependence of the inmedium amplitudes in self-consistent calculations of kaonic nuclei. In Section 3, we present few selected results of our calculations illustrating model dependence and, in particular, the effect of $K^{-}$multinucleon absorption on the widths of $K^{-}$-nuclear quasi-bound states. A brief summary is given in Section 4.

\section{Model}

We determine the binding energies $B_{K^{-}}$and widths $\Gamma_{K^{-}}$of $K^{-}$-nuclear quasi-bound states by solving the Klein-Gordon equation

$$
\left[\vec{\nabla}^{2}+\tilde{\omega}_{K^{-}}^{2}-m_{K^{-}}^{2}-\Pi_{K^{-}}\left(\omega_{K^{-}}, \rho\right)\right] \phi_{K^{-}}=0 .
$$

Here, $\tilde{\omega}_{K^{-}}=m_{K^{-}}-B_{K^{-}}-\mathrm{i} \Gamma_{K^{-}} / 2-V_{C}=\omega_{K^{-}}-V_{C}, m_{K^{-}}$denotes the $K^{-}$mass, $V_{C}$ is the Coulomb potential, and $\rho$ is the nuclear density distribution. The energy- and density-dependent kaon selfenergy operator $\Pi_{K^{-}}$, which describes the $K^{-}$interactions with the nuclear medium, is constructed in a $t \rho$ form as follows:

$$
\Pi_{K^{-}}=2 \operatorname{Re}\left(\omega_{K^{-}}\right) V_{K^{-}}^{(1)}=-4 \pi \frac{\sqrt{s}}{m_{N}}\left(F_{0} \frac{1}{2} \rho_{p}+F_{1}\left(\frac{1}{2} \rho_{p}+\rho_{n}\right)\right)
$$


where $F_{0}$ and $F_{1}$ are the in-medium isospin 0 and $1 s$-wave amplitudes, $V_{K^{-}}^{(1)}$ is the $K^{-}$-nucleus optical potential and $m_{N}$ is the nucleon mass. The kinematical factor $\sqrt{s} / m_{N}$ transforms the scattering amplitudes from the two-body frame to the $K^{-}$-nuclear frame. The realistic proton and neutron density distributions, $\rho_{p}$ and $\rho_{n}$, are taken from the relativistic mean-field model NL-SH [10]. We consider static density distribution, i. e. the core polarization effects are not included [11].

The in-medium scattering amplitudes are obtained from the free-space amplitudes $F_{K^{-}}$and $F_{K^{-}}$, derived within chirally-inspired models of meson-baryon interactions [2, 3, 4, 5], using the multiple scattering approach (WRW) [12] in order to account for Pauli correlations. They are of the following form:

$$
F_{1}=\frac{F_{K^{-} n}(\sqrt{s})}{1+\frac{1}{4} \xi_{k} \frac{\sqrt{s}}{m_{N}} F_{K^{-} n}(\sqrt{s}) \rho}, \quad F_{0}=\frac{\left[2 F_{K^{-}} p(\sqrt{s})-F_{K^{-} n}(\sqrt{s})\right]}{1+\frac{1}{4} \xi_{k} \frac{\sqrt{s}}{m_{N}}\left[2 F_{K^{-}} p(\sqrt{s})-F_{K^{-} n}(\sqrt{s})\right] \rho},
$$

where $\xi_{k}$ is adopted from Ref. [1].

The amplitudes are a function of energy defined by Mandelstam variable

$$
s=\left(E_{N}+E_{K^{-}}\right)^{2}-\left(\vec{p}_{N}+\vec{p}_{K^{-}}\right)^{2},
$$

where $E_{N}=m_{N}-B_{N}, E_{K^{-}}=m_{K^{-}}-B_{K^{-}}$and $\vec{p}_{N\left(K^{-}\right)}$is the nucleon (kaon) momentum. In the $K^{-}$nucleus frame the momentum dependent term is no-longer zero and provides additional downward energy shift. The energy shift $\delta \sqrt{s}=\sqrt{s}-E_{t h}$ can be expanded in terms of binding and kinetic energies as follows [13]:

$$
\delta \sqrt{s}=-B_{N} \frac{\rho}{\bar{\rho}}-\beta_{N}\left[B_{K^{-}} \frac{\rho}{\rho_{\max }}+T_{N}\left(\frac{\rho}{\bar{\rho}}\right)^{2 / 3}+V_{C}\left(\frac{\rho}{\rho_{\max }}\right)^{1 / 3}\right]+\beta_{K^{-}} \operatorname{Re} V_{K^{-}}(r),
$$

where $B_{N}=8.5 \mathrm{MeV}$ is the average binding energy per nucleon, $\bar{\rho}$ is the average nuclear density, $\rho_{\max }$ is the maximal value of the nuclear density, $\beta_{N\left(K^{-}\right)}=m_{N\left(K^{-}\right)} /\left(m_{N}+m_{K^{-}}\right)$and $T_{N}=23 \mathrm{MeV}$ is the average nucleon kinetic energy in the Fermi Gas Model. The energy shift respects the lowdensity limit, i. e. $\delta \sqrt{s} \rightarrow 0$ as $\rho \rightarrow 0$ and the minimal substitution requirement $E \rightarrow E-V_{C}$ [14].

The above chiral models describe only the $K^{-}$interactions with a single nucleon. However, inside the medium, the $K^{-}$interactions with two and more nucleons take place and should be considered as well [7, 15]. Therefore, we supplement the $K^{-}$single-nucleon potential $V_{K^{-}}^{(1)}$ from Eq. (2.2) with a phenomenological optical potential $V_{K^{-}}^{(2)}$ describing the $K^{-}$multinucleon interactions of the form

$$
2 \operatorname{Re}\left(\omega_{K^{-}}\right) V_{K^{-}}^{(2)}=-4 \pi B\left(\frac{\rho}{\rho_{0}}\right)^{\alpha} \rho .
$$

The parameters of the phenomenological potential, complex amplitude $B$ and positive exponent $\alpha$, were recently fitted by Friedman and Gal [1] to kaonic atom data for each chirally-motivated meson-baryon interaction model considered in this work separately. Moreover, the total $K^{-}$potential, $V_{K^{-}}=V_{K^{-}}^{(1)}+V_{K^{-}}^{(2)}$, was confronted with branching ratios of $K^{-}$absorption at rest from bubble chamber experiments $[16,17,18]$ and only the P and KM models were found capable of reproducing both experimental constraints simultaneously.

The kaonic atom data probe the $K^{-}$optical potential up to at most $\sim 50 \%$ of the nuclear density [1]. Further inside the nucleus, the shape of the potential is just an analytical continuation 
of the formula. Therefore, we first apply the full formula of Eq. (2.6) (full density option - FD) in the calculations and then we fix the potential $V_{K^{-}}^{(2)}$ at constant value $V_{K^{-}}^{(2)}\left(0.5 \rho_{0}\right)$ for $\rho(r) \geq 0.5 \rho_{0}$ (half density limit - HD).

\section{Results}

The formalism outlined in the previous section was applied to self-consistent calculations of $K^{-}$quasi-bound states in nuclei across the periodic table. First, the predictions for binding energies and corresponding widths of $K^{-}$-nuclear quasi-bound states calculated within the 6 different chiral models were compared. Then the $K^{-}$single-nucleon potential derived from the $\mathrm{P}$ and $\mathrm{KM}$ models was supplemented by the phenomenological $K^{-}$multinucleon potential and the impact of $K^{-}$multinucleon absorption on $K^{-}$widths was explored.

In the left panels of Fig. 1, we present the free-space $s$-wave $K^{-} p$ (upper block) and $K^{-} n$ (lower block) scattering amplitudes in all models considered. The $F_{K^{-}} p$ amplitudes agree with each other (except Bonn models B2 and B4 ${ }^{1}$ ) at and above threshold since the parameters of the models were fitted to experimental data in this region. However, the $K^{-} p$ amplitudes differ considerably below threshold - in the region relevant for $K^{-}$-nuclear states calculations. Moreover, the amplitudes are strongly energy dependent due to the nearby resonance $\Lambda(1405)$. The B2 and B4 models predict even repulsive real part of the $K^{-} p$ amplitude. The $K^{-} n$ amplitudes do not match each other even at and above threshold. In Bonn models, the real part of the $K^{-} n$ amplitudes is repulsive again. Based on the very different energy dependence of the $K^{-} N$ scattering amplitudes below threshold we can expect large variety in the predictions of the binding energies and widths of $K^{-}$-nuclear states.

The in-medium $K^{-} p$ and $K^{-} n$ amplitudes at saturation density $\rho_{0}=0.17 \mathrm{fm}^{-3}$ are shown in the right panels of Fig. 1. The real parts of the $K^{-} p$ amplitudes are affected considerably by the Pauli correlations - they become attractive in the entire energy region (except the Bonn models B2 and B4). The peaks of the $K^{-} p$ imaginary in-medium amplitudes are significantly lowered below threshold. On the contrary, the $K^{-} n$ amplitudes remain almost intact by the medium modifications (lower block, right panels).

In Fig. 2, $1 s K^{-}$binding energies (left panel) and corresponding widths (right panel) are shown as a function of mass number $A$, calculated in various nuclei using the $K^{-}$single-nucleon potential derived within different chiral meson-baryon interaction models. The binding energies $B_{K^{-}}$exhibit the same $A$ dependence in all models considered, however, their magnitudes are strongly model dependent. It is due to the different depths of $\operatorname{Re}_{K^{-}}^{(1)}$ predicted by the $K^{-} N$ interaction models. The $K^{-} N$ conversion widths are rather small and weakly $A$-dependent. The KM model predicts widths twice as large as the P and M1 models. We did not succeed to obtain any bound state for the Bonn models since the real parts of the corresponding $K^{-} N$ scattering amplitudes are repulsive below threshold.

In Fig. 3, we present the $1 s K^{-}$binding energies and widths in various nuclei, calculated selfconsistently in the KM model and HD and FD versions of the $K^{-}$multinucleon potential with $\alpha=1$ (KM1). The binding energies and corresponding widths calculated with underlying chirallyinspired $K^{-}$single-nucleon potential ( $\mathrm{KN}$, green dots) are shown for comparison. Adding the

\footnotetext{
${ }^{1}$ The B2 and B4 amplitudes differ from the others since higher partial waves were included in the Bonn models fits.
} 

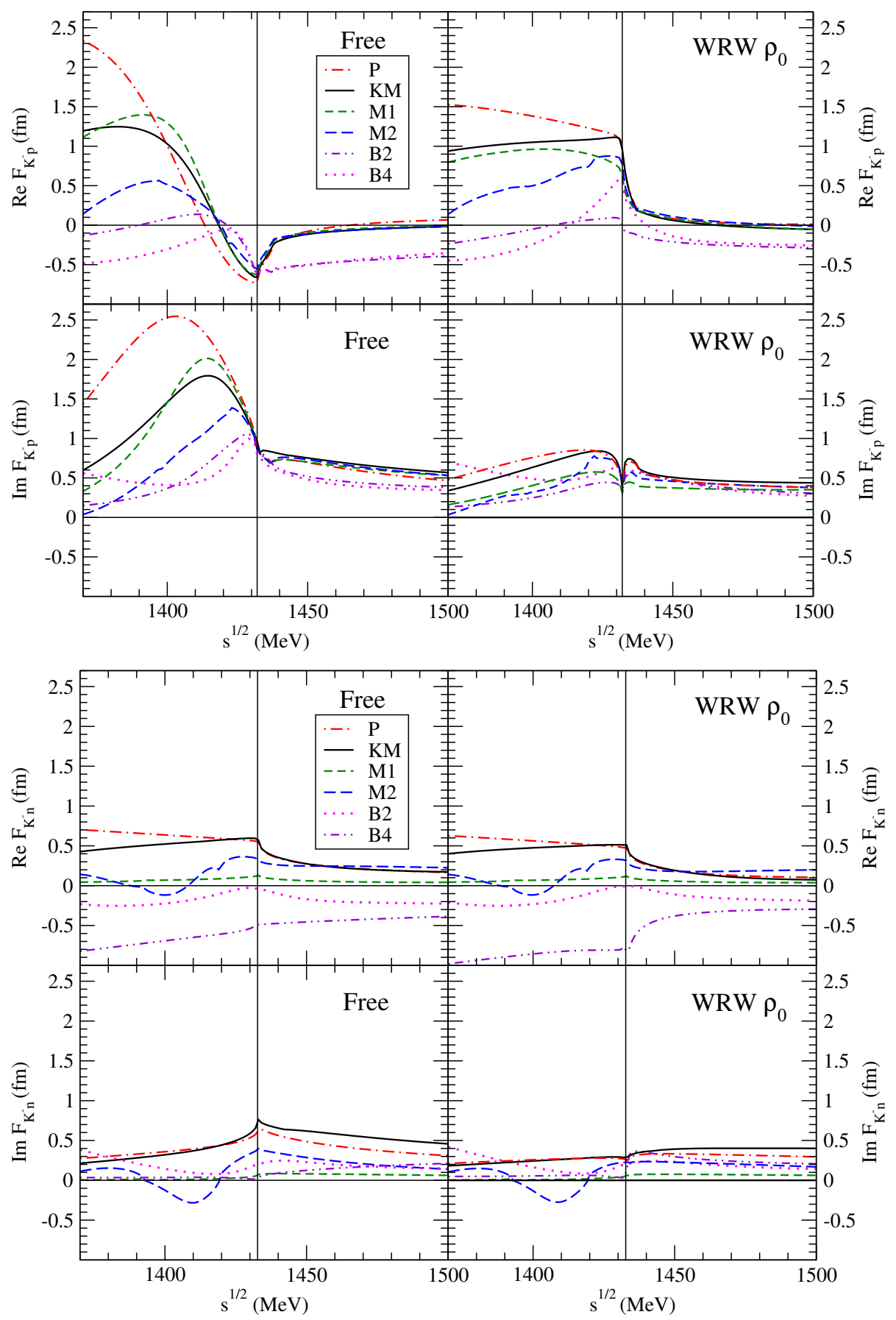

Figure 1: Energy dependence of free-space amplitudes $K^{-} p$ (upper block, left panels) and $K^{-} n$ (bottom block, left panels), compared with corresponding WRW modified in-medium amplitudes (right panels) for $\rho_{0}=0.17 \mathrm{fm}^{-3}$ in considered models.

$K^{-}$multinucleon potential to the $K^{-}$single-nucleon potential affects significantly the $K^{-}$widths. Within the HD version the widths are twice as large as in the KN case. Consequently, the $K^{-}$ binding energies are lower as well but the change is not so pronounced $(<20 \%)$. The FD version 

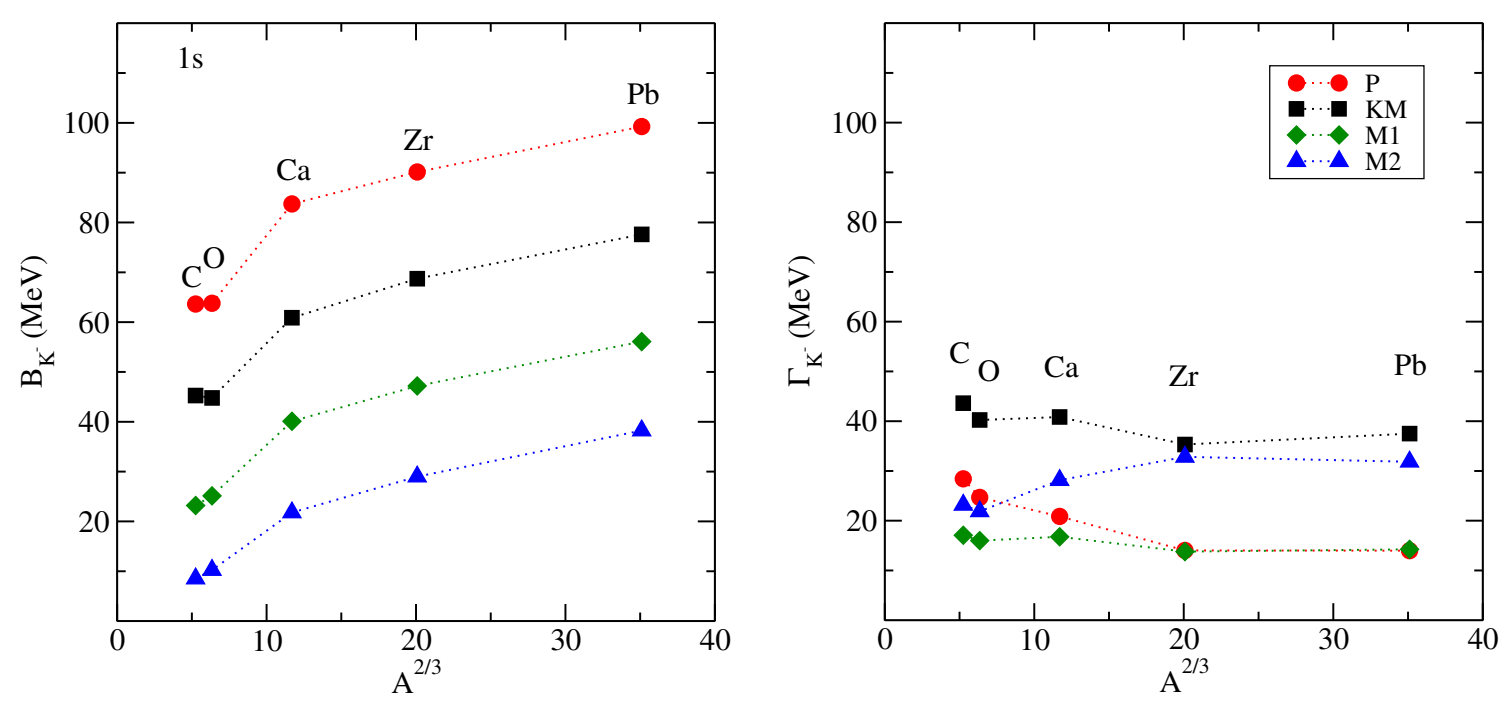

Figure 2: $1 s \mathrm{~K}^{-}$binding energies (left) and corresponding widths (right) in various nuclei calculated selfconsistently in the P, KM, M1, and M2 models.
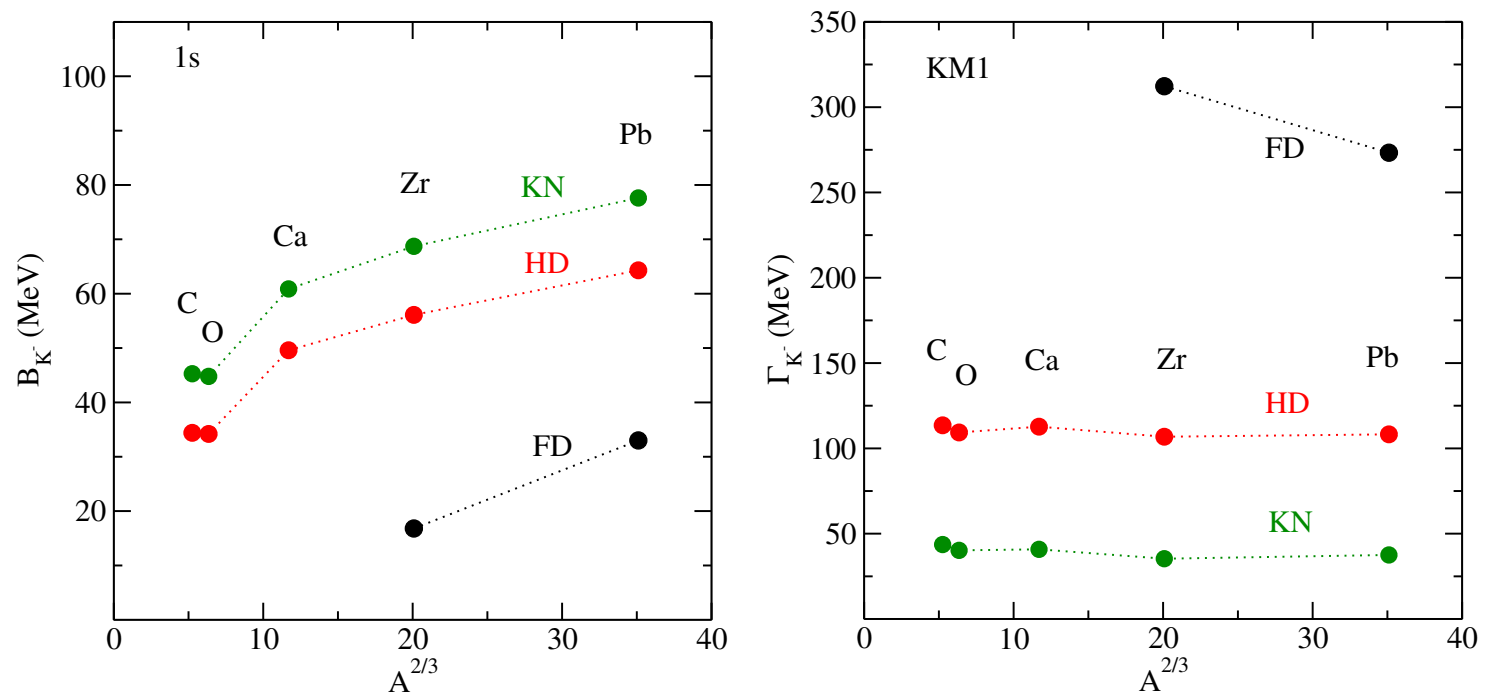

Figure 3: $1 s K^{-}$binding energies $B_{K^{-}}$(left panel) and corresponding widths $\Gamma_{K^{-}}$(right panel) as a function of mass number $A$, calculated with the HD and FD versions of the $K^{-}$multinucleon potential in the KM1 model (see text for details). The $K^{-}$binding energies and widths calculated with the $K^{-}$single-nucleon potential are shown for comparison (KN, green dots).

of the multinucleon potential does not even yield any $K^{-}$bound states in nuclei with $A \leq 40$. We found $1 s K^{-}$quasi-bound states in ${ }^{90} \mathrm{Zr}$ and ${ }^{208} \mathrm{~Pb}$, however, the binding energies of these states are small and the corresponding widths are huge — one order of magnitude larger than the binding energies. Similar results were obtained in the KM model with $\alpha=2$ (KM2) and the P model with $\alpha=1$ and 2 (P1 and P2) as well. We thus dare to claim that these results are valid generally.

Finally, we evaluated the fractions of $K^{-}$single-nucleon and multinucleon absorption in the 


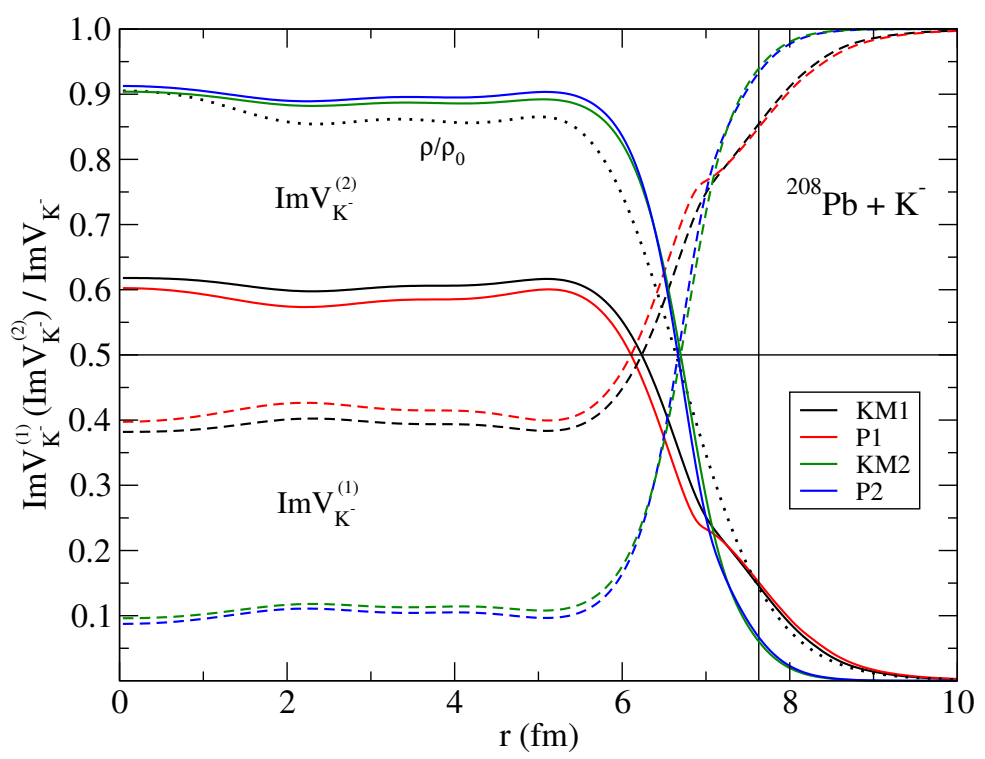

Figure 4: The ratio of $\operatorname{Im} V_{K^{-}}^{(1)}$ (dashed line) and $\operatorname{Im} V_{K^{-}}^{(2)}$ (solid line) potentials to the total $K^{-}$imaginary potential $\operatorname{Im} V_{K^{-}}$as a function of radius in ${ }^{208} \mathrm{~Pb}+K^{-}$system, calculated self-consistently for the FD version of $K^{-}$multinucleon potential in the KM and P models. The relative density $\rho / \rho_{0}$ (dotted line) is shown for comparison.

medium as ratios of $\operatorname{Im} V_{K^{-}}^{(1)}$ and $\operatorname{Im} V_{K^{-}}^{(2)}$ with respect to the total imaginary $K^{-}$potential $\operatorname{Im} V_{K^{-}}$. They are shown in Fig. 4 as a function of radius, calculated self-consistently for ${ }^{208} \mathrm{~Pb}$, using FD option of $V_{K^{-}}^{(2)}$ in the KM1(2) and P1(2) models. The relative density $\rho / \rho_{0}$ is shown for comparison (dotted line). The $K^{-}$absorption fractions are changing with radius (and thus with density). At the surface of the nucleus, the $K^{-}$absorption on a single nucleon dominates. Friedman and Gal [1] showed that the fractions of $K^{-}$absorption on a single-nucleon $(\sim 75 \%)$ and several nucleons $(\sim 25 \%)$ from bubble chamber experiments are sensitive to about $15 \%$ of nuclear density (denoted by vertical black line). At this value of density, the calculated fractions are slightly different from experimental ones due to different values of $\sqrt{s}$ for kaonic and nuclear states. Inside the nucleus, the $K^{-}$single-nucleon absorption is suppressed due to the vicinity of $\pi \Sigma$ threshold and the multinucleon absorption prevails. The ratios in the KM1 and P1 models differ from those in the KM2 and P2 models due to different self-consistent values of the energy shift $\delta \sqrt{s}$ below threshold and the different density dependence of the $K^{-}$multinucleon potentials $V_{K^{-}}^{(2)}(2.6)$.

\section{Summary}

In this contribution, we presented our self-consistent calculations of $K^{-}$-nuclear quasi-bound states using $K^{-}$single-nucleon potentials derived from 6 different chirally-motivated meson-baryon interaction models. We showed that the resulting $K^{-}$binding energies are strongly model dependent and the corresponding $K^{-} N \rightarrow \pi Y(Y=\Lambda, \Sigma)$ decay widths are rather small. Then we supplemented the $K^{-}$single-nucleon potential derived within the $\mathrm{P}$ and $\mathrm{KM}$ models by a phenomenological optical potential describing the $K^{-}$multinucleon interactions. Parameters of the 
phenomenological potential were recently fitted to kaonic atom data for each of the chiral models separately [1]. We demonstrated that the $K^{-}$multinucleon absorption increases the $K^{-}$widths drastically. The widths of $K^{-}$-nuclear states are one order of magnitude larger than the corresponding binding energies, if there is any bound state in the particular nucleus at all.

\section{Acknowledgments}

We thank E. Friedman and A. Gal for valuable discussions. J. H. wishes to thank the organizers for providing her with the student fellowship. This work was supported by GACR Grant No. P203/15/04301S.

\section{References}

[1] E. Friedman, A. Gal, Nucl. Phys. A 959 (2017) 66.

[2] A. Cieplý, J. Smejkal, Nucl. Phys. A 881 (2012) 115.

[3] Y. Ikeda, T. Hyodo, W. Weise, Nucl. Phys. A 881 (2012) 98.

[4] Z. H. Guo, J. A. Oller, Phys. Rev. C 87 (2013) 035202.

[5] M. Mai and U.-G. Meißner, Nucl. Phys. A 900 (2013) 51.

[6] M. Bazzi et al (SIDDHARTA Collaboration), Phys. Lett. B 704 (2011) 113.

[7] J. Mareš, E. Friedman, A. Gal, Phys. Lett. B 606 (2005) 295.

[8] J. Hrtánková, J. Mareš, Phys. Lett. B in print, arXiv:1703.01788 [nucl-th].

[9] J. Hrtánková, J. Mareš, subm. Phys. Rev. C, arXiv:1704.07205 [nucl-th].

[10] M. M. Sharma, M. A. Nagarajan, P. Ring, Phys. Lett. B 312 (1993) 377.

[11] D. Gazda, J. Mareš, Nucl. Phys. A 881 (2012) 159.

[12] T. Wass, M. Rho, W. Weise, Nucl. Phys. A 617 (1997) 449.

[13] J. Hrtánková, J. Mareš, Nucl. Phys. A 945 (2016) 197.

[14] E. E. Kolomeitsev, N. Kaiser, W. Weise, Phys. Rev. Lett. 90 (2003) 092501.

[15] C. J. Batty, E. Friedman, A. Gal, Phys. Rept. 287 (1997) 385.

[16] H. Davis et al., Nuovo Cimento 53 A (1968) 313.

[17] J. W. Moulder et al., Nucl. Phys. B 35 (1971) 332.

[18] C. Vander Velde-Wilquet et al., Nuovo Cimento 39 A (1977) 539. 